\title{
Life Cycle Assessment of Bioethanol Production: A Review of Feedstock, Technology and Methodology
}

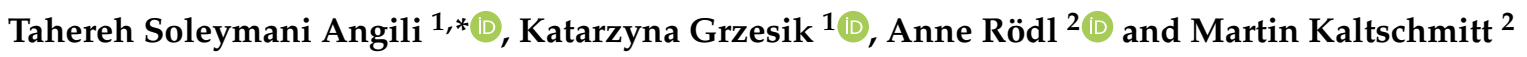 \\ 1 Faculty of Mining Surveying and Environmental Engineering, AGH University of Science and Technology, \\ Mickiewicza Av. 30, 30-059 Krakow, Poland; grzesikk@agh.edu.pl \\ 2 Institute of Environmental Technology and Energy Economics (IUE), Hamburg University of \\ Technology (TUHH), Eissendorfer Str. 40, 21073 Hamburg, Germany; anne.roedl@tuhh.de (A.R.); \\ kaltschmitt@tuhh.de (M.K.) \\ * Correspondence: angili@agh.edu.pl
}

check for updates

Citation: Soleymani Angili, T.;

Grzesik, K.; Rödl, A.; Kaltschmitt, M. Life Cycle Assessment of Bioethanol Production: A Review of Feedstock, Technology and Methodology. Energies 2021, 14, 2939.

https://doi.org/10.3390/en14102939

Academic Editor: Sergio Ulgiati

Received: 18 April 2021

Accepted: 16 May 2021

Published: 19 May 2021

Publisher's Note: MDPI stays neutral with regard to jurisdictional claims in published maps and institutional affiliations.

Copyright: (c) 2021 by the authors. Licensee MDPI, Basel, Switzerland. This article is an open access article distributed under the terms and conditions of the Creative Commons Attribution (CC BY) license (https:/ / creativecommons.org/licenses/by/ $4.0 /)$.

\begin{abstract}
So far, a lot of efforts have been put in life cycle assessments (LCA) of bioethanol production. There are many works that have assessed bioethanol production in different points of view to illustrate the environmental impacts. This study reviewed former LCA studies on bioethanol produced from various biomass resources by considering the effect of methodological components, technical pathways and feedstock provision on the result of LCA studies. The review evaluated 48 papers published 2002-2021 with a focus on studies that included a complete set of environmental impact categories. However, due to lack of harmony among studies, comparing the LCA results was challenging but the review indicated that the final results of studies are influenced by LCA methodological components, such as system boundary, functional unit, etc. Around $80 \%$ of the reviewed papers show the reduction in global warming potential, while contrary results have been found about increasing acidification, eutrophication and photochemical oxidant formation impact categories because of the feedstock provision. Regarding technical aspects, results from the review revealed that most of the studies considered the pre-treatment as a crucial step in bioconversion processes. Despite several LCA studies of bioethanol production, there is still low attention given to uncertainty analysis in the publications.
\end{abstract}

Keywords: LCA; biofuel; methodological components; feedstock provision; technical aspects

\section{Introduction}

Over the past few years, the policy context for renewable energy has changed considerably [1]. In this regard, international institutions help the countries to set the necessary targets towards a higher renewables' deployment. Renewable Energy Directive (RED) 2018/2001 of the European Parliament addressed energy generation from renewable sources in member countries until 2030 [2]. Further, organizations such as REN21: Renewables Now! International Renewable Energy Agency and International Energy Agency by providing up-to-date information support decision-makers from politics and industry for a sustainable renewable energy future.

Amongst the targets and strategy setting for renewable energies, the development of biofuels, including bioethanol, has also been considered. Bioethanol is the main renewable energy carrier after biodiesel in Europe [3] that can be produced from a variety of biomass feedstock, including, e.g., energy crops and lignocellulosic biomass. The United States is the world's largest producer of bioethanol. Brazil and the European Union are ranked in the second and third place, respectively [4]. The vast majority of U.S. bioethanol is produced from corn, while Brazil primarily uses sugarcane [4], and corn is the main crop for bioethanol production in Europe [5]. The substitution of fossil fuels started with ethanol produced from food crops containing starch or sugar as the first-generation bioethanol. However, the conversion of food crops into bioethanol raised concerns about food security 
on a global scale $[6,7]$. Therefore, a second-generation of bioethanol based on non-food resources like lignocelluloses is under development. The following sections will present a summary of bioethanol production from various sources.

- Starch based bioethanol

Crops such as corn, wheat or barley are known as starch biomass with the purpose of producing ethanol. Moreover, other kind of starchy crops like cassava in tropical areas can be used for production of ethanol. In starchy materials polymers of glucose require to break down to fermentable sugars through a reaction with water and adding enzymes. Then enzymatic hydrolysis is followed by fermentation, distillation and dehydration to yield anhydrous ethanol.

- Sugar based bioethanol

Sugar-based feedstock such as sugarcane, sugar beets, sweet sorghum and molasses are potential raw materials used for ethanol production. They contain a large proportion of simple sugars that leads to cost reduction of conversion processes. Conversion starts by feedstock grinding to extract the sugar, then is followed by adding yeast for the fermentation process.

- $\quad$ Lignocellulose based bioethanol

Lignocellulosic materials, including agricultural and forestry residues, are abundant all over the world. They can be used to produce ethanol as second-generation bioethanol. Lignocellulose is a more complex substrate than sugar and starch-based materials. It is composed of three fractions, including cellulose, hemicellulose and lignin. Conversion of carbohydrate polymer to fermentable sugars is not easy since ethanol production from lignocellulosic biomass is a multi-stage conversion.

The most important reasons for bioethanol's widespread deployment are environmental challenges caused by fossil fuels and their depletion [6,8]. It is expected that switching to renewable sources like bioethanol reduces impacts on the environment. However, its production may have several impacts depending on feedstock type, local conditions, design and implementation of the respective conversion process [9]. Hence, to identify the environmental burdens of the bioethanol production system, a comprehensive evaluation is required. Life cycle assessment, defined according to ISO14040 and 14044 [9,10], is mainly used to identify and measure the environmental impact towards sustainability [11]. Several LCA studies have been carried out to determine savings in energy and emissions throughout the life cycle of bioethanol production.

The main aim of this paper is an overview of previous LCA studies for bioethanol production, followed by results on methodological and technological issues related to crop production based on the following sections. Moreover, the purpose of this review is to recognize bottlenecks and improve future research works by investigating the influence of different parameters on the final results of LCA.

\section{Method}

\subsection{Approach of Review}

In recent years, many LCA studies of bioethanol production were conducted. Each of them used different objectives, various feedstock, varying technological approaches, and different methodological components. Therefore, comparing their results and finding the best practice is a challenging task. In the first step of the overview, records were identified searching through Google Scholar, ResearchGate and SCOPUS.

The search approach was conducted on joining two key expressions: "life cycle assessment" and "bioethanol production". After removing the duplicated and unrelated items, the papers not meeting the inclusion/exclusion criteria were removed at this stage. Then, full-text assessment started to check eligibility. Papers were selected when they revealed LCA was carried out, feedstock provided from $1 \mathrm{G}$ and $2 \mathrm{G}$ agro-based substrates, a set of environmental impact categories was analyzed, as well as the biochemical processes 
used in the biorefinery. Finally, eligible studies were included in a qualitative analysis of the effect of feedstock provision and conversion processes on LCA results, as well as methodological choices.

\subsection{Data Description}

The screening process classified 48 suitable studies out of 66 papers (as explained in Section 2.1) published between 2002 and 2021. The papers include 34 LCA studies on bioethanol from second-generation feedstock, 11 on bioethanol of first-generation feedstock. The remaining examined both types of resources.

Those studies that assessed only a single impact category or energy usage or, feedstock from non-agro residues or thermochemical conversion process are not included in this review. Bioethanol LCA studies have been conducted mainly by European countries since 2009. However, in recent years gradually the scope of the published studies has expanded to include Asia and South / North America. More than $50 \%$ of the papers assessed here compared the results with a reference case often being gasoline production, another feedstock type, or a different conversion process.

\section{Results and Discussion}

\subsection{Technical Aspects}

The biochemical conversion of biomass feedstock comprises the following main steps:

- Feedstock handling and pre-treatment;

- Saccharification to reach fermentable sugars;

- Fermentation process to convert sugars to ethanol;

- Ethanol recovery.

Figure 1 shows the scheme of production processes for 1G biomass conversion to ethanol. Although the mentioned processes are fundamental in bioconversion, each type of feedstock requires a special conversion method. All the above-mentioned processes require energy, water and chemicals. Therefore, conversion technologies are important sources of environmental impact during bioethanol production.

The pre-treatment stage in bioconversion plays a key role in influencing downstream processes [12]. Choosing a suitable pre-treatment method can change the following processes and LCA results. Shadbahr et al. [13] noted that modification of pre-treatment by increasing the concentration of sulfuric acid and decreasing residence time in the reactor improves environmental impacts. Moreover, the effect of the type of pre-treatment process on impact categories has been argued by Guo et al. [14]. Here, dilute acid and liquid hot water pre-treatment methods were examined. According to the results, the dilute acid method caused higher impacts on acidification potential, eutrophication potential, and ecotoxicity because of more chemical consumption whilst the latter increased global warming potential, abiotic depletion and ozone depletion potential due to more enzyme usage as an energy-intensive product.

In another study by Wang et al. [15], different types of pre-treatment methods, including dilute acid, steam explosion, liquid hot water and wet oxidation, are compared. The result reveals that dilute acid increased all impact categories of CML baseline 2000 method whereas steam explosion pre-treatment caused the lowest environmental burdens in all categories.

Ntihuga et al. [16] compared two scenarios of bioethanol production by continuous fermentation Blenke cascade system with and without co-product utilization. Results showed the scenario with yeast and $\mathrm{CO}_{2}$ recycling, as well as double saccharification processes contributes to low impacts in all categories of CML 2010 method. 


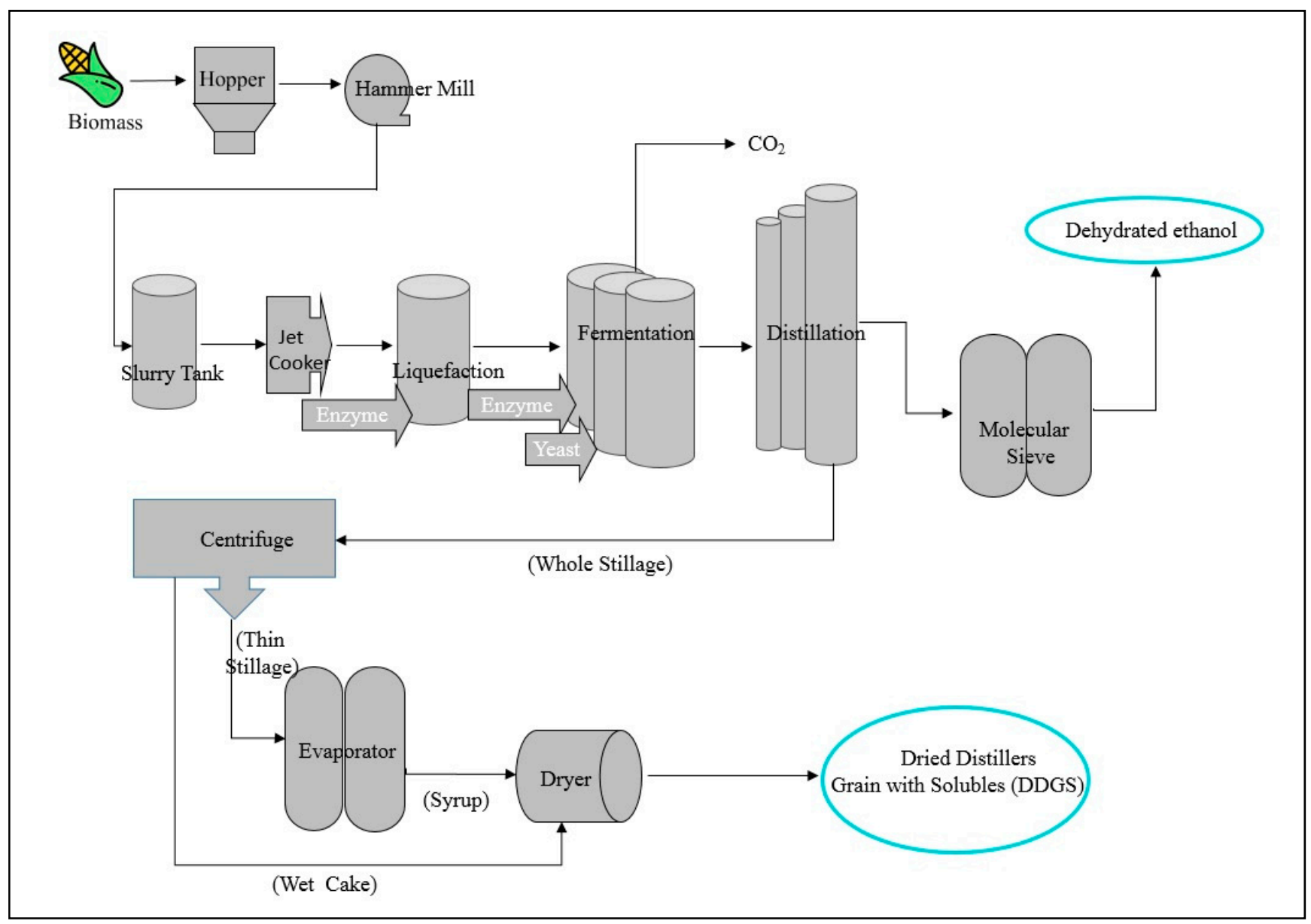

Figure 1. Schematic diagram of bioethanol production for first generation feedstock.

\subsection{Feedstock Provision}

The type of feedstock used for ethanol production determines the conversion processes or the agricultural measures to prepare the raw material, which is reflected in the life cycle assessment results. For instance, lignin-rich materials, such as wood, need an efficient pre-treatment process which results in higher energy consumption for the conversion of the biomass into bioethanol [13].

Most of the reviewed studies (46 papers) integrated the crop cultivation in their system boundaries. Figure 2 shows the type of biomass studied in the reviewed papers. These papers included all activities for feedstock preparation and transport. They investigated the effect of planting and fertilizing, residues utilization on LCA results, especially on the impact category land-use change. Besides others, it was found that using substrates from naturally occurring species would decrease LUC. Research conducted by Falano et al. [17] revealed that land use shifting from forest area to other species like Miscanthus increases GHG emissions from the soil whilst forest cultivation instead of grass leads to reverse results. 


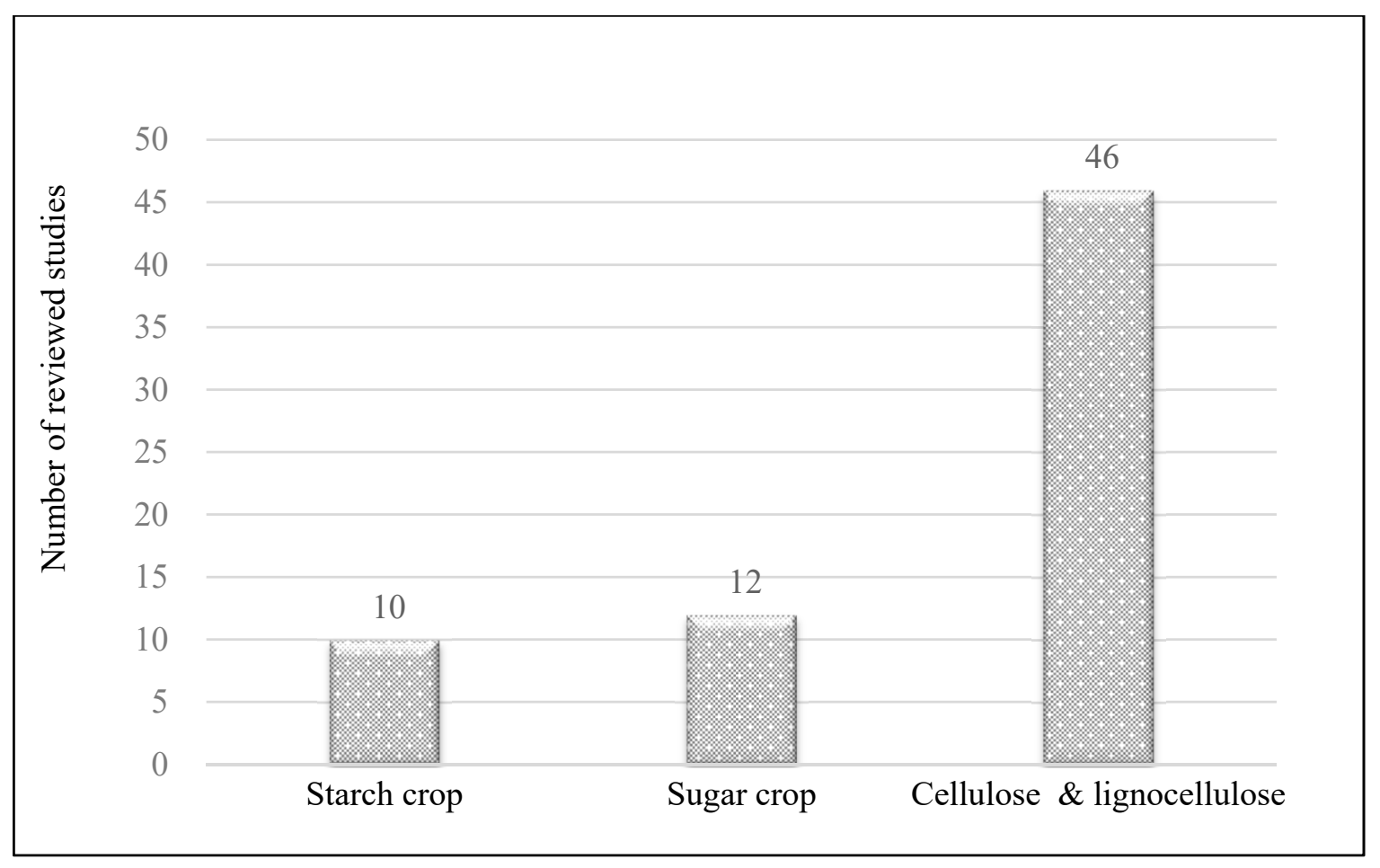

Figure 2. Type of biomass considered by reviewed papers (some of papers studied more than one feedstock).

Further, using alternative feedstock like residues, by-products or new species showed changes in environmental impacts [18]. One of the reviewed studies showed that residual materials have less contribution to land-use change in comparison to main crops due to joint production and reduced use of chemicals, water and fuel [19]. One of the papers that advanced practices like genetic improvements and developed breeding programs can influence the effectiveness of ethanol production [14]. The biomass type also determines the kind and number of agrochemicals that are used, which also influence the LCA results.

Among the LCA studies reviewed here, 21 included agrochemicals production in the system boundary. Their result showed that the use of chemicals, such as fertilizers and pesticides, increases acidification, eutrophication, land-use change and carcinogens.

\subsection{LCA Methodology Components}

\subsubsection{System Boundary and Functional Unit}

The system boundary is setting the frame for the assessed process. It also includes different variables, such as data availability, purpose of study, time, and budget. The boundary for the assessed bioethanol production systems ideally could be defined as cradle-grave covering all processes during a life span. Figure 3 shows a general overview of the processes and system boundaries in the reviewed papers.

The most widely used boundaries by the reviewed studies were cradle-gate ( 25 case studies), followed by cradle-grave (22 cases) and three cases of gate-to-gate type. Table 1 shows the studies that examined the LCA for bioethanol production. 


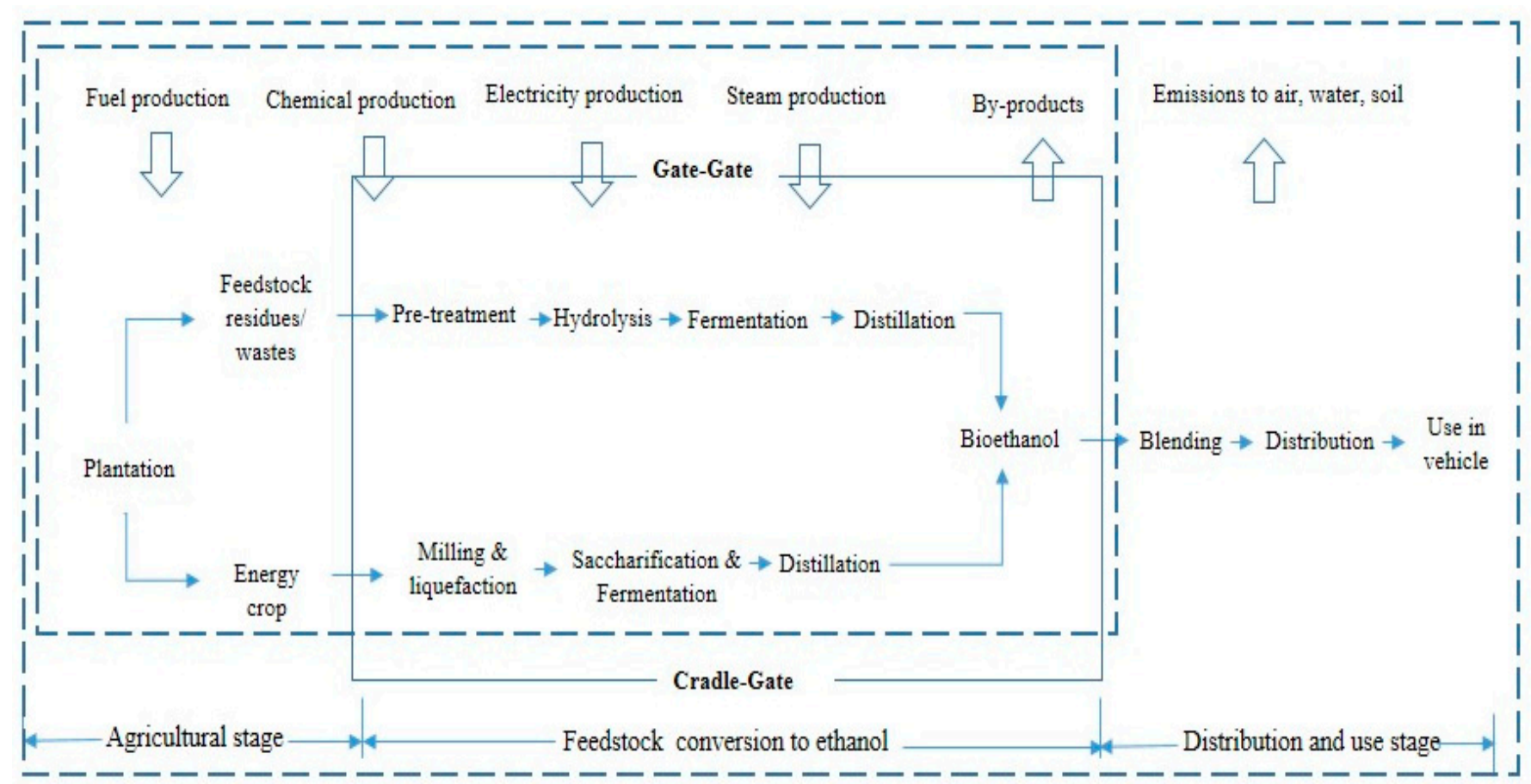

Cradle-Grave

Figure 3. An overview of system boundaries and technological process of $1 \mathrm{G}$ and $2 \mathrm{G}$ bioethanol considered by reviewed papers. 
Table 1. Summary of the reviewed publications. $(\mathrm{ND}=$ not detected $)$.

\begin{tabular}{|c|c|c|c|c|c|c|c|c|}
\hline $\begin{array}{l}\text { Reference/Authors and } \\
\text { Reference Number as } \\
\text { Required by Energies }\end{array}$ & Biomass Source & Location & System Boundary & Functional Unit & $\begin{array}{l}\text { Data Source/Type of } \\
\text { Collected Data }\end{array}$ & Software & LCIA Method/Type & Allocation Method \\
\hline [20] & Sweet potato & China & Cradle-gate & Ethanol product & Industry, GREET & ND & CML & ND \\
\hline [21] & Sugarcane & Brazil & Cradle-gate & Ethanol product & Industry & SimaPro & Ecoindicator & Mass, energy \\
\hline [16] & Wheat & Germany & Cradle-gate & Ethanol product & $\begin{array}{l}\text { Experimental data, } \\
\text { Lean database }\end{array}$ & $\mathrm{GaBi}$ & CML & ND \\
\hline [22] & Corn grain & USA & Cradle-grave & Ethanol product & $\begin{array}{l}\text { GREET, NREL, } \\
\text { DEAM database }\end{array}$ & ND & TRACI, IPCC & System expansion \\
\hline [23] & Sugarcane & Brazil & Cradle-grave & Caloric value & $\begin{array}{l}\text { Ecoinvent, process } \\
\text { simulation }\end{array}$ & SimaPro & $\begin{array}{l}\text { TRACI, EDIP, } \\
\text { IMPACT 2002, } \\
\text { Ecological scarcity } \\
\text { 2006, ReCiPe, CML, } \\
\text { Ecoindicator }\end{array}$ & Energy \\
\hline [24] & Wheat, sugar beet & Germany & Cradle-gate & Caloric value & Industry, ecoinvent & Umberto & ReCiPe, CED & Energy \\
\hline [19] & Corn grain & Argentina & Cradle-gate & Caloric value & $\begin{array}{l}\text { Industry, ecoinvent, } \\
\text { NREL }\end{array}$ & SimaPro & ReCiPe, Ecoindicator & Economic \\
\hline [25] & Sugarcane & Brazil & Cradle-grave & Driving distance & $\begin{array}{c}\text { PestLCI software, } \\
\text { industry, simapro } \\
\text { databases }\end{array}$ & SimaPro & EDIP & Energy \\
\hline [26] & Sugarcane & Brazil & Cradle-grave & Driving distance & $\begin{array}{c}\text { Ecoinvent, EIPRO } \\
\text { database, }\end{array}$ & CMLCA & CML & Economic \\
\hline [28] & Sugar beet & Greece & Cradle-gate & Caloric value & Industry, ecoinvent & SimaPro & CML & $\begin{array}{c}\text { ND } \\
\text { System expansion. }\end{array}$ \\
\hline $\begin{array}{l}{[14]} \\
{[29]}\end{array}$ & $\begin{array}{c}\text { Poplar } \\
\text { Sweet sorghum }\end{array}$ & $\begin{array}{c}\text { Sweden, France, } \\
\text { Italy, Spain, Slovakia } \\
\text { China }\end{array}$ & $\begin{array}{l}\text { Cradle-grave } \\
\text { Cradle-gate }\end{array}$ & $\begin{array}{l}\text { Driving distance } \\
\text { Ethanol product }\end{array}$ & $\begin{array}{c}\text { Aspen Plus } \\
\text { GREET pilot plant }\end{array}$ & $\begin{array}{l}\text { SimaPro } \\
\text { ND }\end{array}$ & $\begin{array}{l}\text { CML, Ecoindicator } \\
\text { CML }\end{array}$ & $\begin{array}{c}\text { System expansion, } \\
\text { energy } \\
\text { Energy, } \\
\text { economic }\end{array}$ \\
\hline [30] & Wheat straw & UK & Cradle-grave & $\begin{array}{l}\text { Driving distance, } \\
\text { ethanol product }\end{array}$ & $\begin{array}{l}\text { Laboratory data, } \\
\text { NREL }\end{array}$ & SimaPro & ReCiPe & Mass \\
\hline [31] & Sweet sorghum & Mexico & Cradle-grave & Input biomass & $\begin{array}{c}\text { Ecoinvent, Aspen } \\
\text { Plus }\end{array}$ & SimaPro & CML & System expansion \\
\hline [32] & Corn stover & Turkey & Cradle-grave & Driving distance & Ecoinvent, NREL & $\mathrm{GaBi}$ & IPCC, EDIP & ND \\
\hline [33] & Willow & USA & Cradle-grave & Caloric value & $\begin{array}{l}\text { GREET, Aspen Plus, } \\
\text { NREL, ecoinvent }\end{array}$ & SimaPro & TRACI & Energy \\
\hline [34] & Switchgrass & Austria & Gate-gate & Input biomass & Ecoinvent & SimaPro & CML, CED & ND \\
\hline [13] & Woodchips & Canada & Gate-gate & Ethanol product & NREL, ecoinvent & SimaPro & Ecoindicator & ND \\
\hline [35] & $\begin{array}{l}\text { Corn stover, } \\
\text { switchgrass }\end{array}$ & USA & Cradle-gate & Caloric value & $\begin{array}{l}\text { GREET, ecoinvent, } \\
\text { GaBi }\end{array}$ & ND & IPCC & ND \\
\hline [36] & $\begin{array}{l}\text { Corn stover, wheat } \\
\text { straw }\end{array}$ & Austria & Cradle-gate & Input biomass & $\begin{array}{c}\text { Ecoinvent, ETH-ESU } \\
96\end{array}$ & SimaPro & CML & ND \\
\hline [37] & Poplar & Spain & Cradle-grave & Driving distance & $\begin{array}{c}\text { NREL, Experimental } \\
\text { data }\end{array}$ & ND & CML & ND \\
\hline
\end{tabular}


Table 1. Cont.

\begin{tabular}{|c|c|c|c|c|c|c|c|c|}
\hline $\begin{array}{l}\text { Reference/Authors and } \\
\text { Reference Number as } \\
\text { Required by Energies }\end{array}$ & Biomass Source & Location & System Boundary & Functional Unit & $\begin{array}{c}\text { Data Source/Type of } \\
\text { Collected Data }\end{array}$ & Software & LCIA Method/Type & Allocation Method \\
\hline [7] & Corn stover & Netherlands & Cradle-grave & Driving distance & $\begin{array}{c}\text { NREL, EIPRO } \\
\text { database, ecoinvent }\end{array}$ & CMLCA & CML & $\begin{array}{l}\text { Energy, mass, } \\
\text { economic }\end{array}$ \\
\hline [18] & $\begin{array}{c}\text { Black locust, eucalyptus } \\
\text { and poplar }\end{array}$ & Spain, Italy & Cradle-grave & Driving distance & $\begin{array}{l}\text { NREL, on-site survey, } \\
\text { ecoinvent }\end{array}$ & CMLCA & CML & ND \\
\hline [8] & $\begin{array}{c}\text { Alfalfa, Ethiopian } \\
\text { mustard, flax shive, } \\
\text { hemp hurds and poplar }\end{array}$ & Spain & Cradle-grave & Driving distance & NREL, ecoinvent & ND & CML & Mass \\
\hline [15] & Wheat straw & UK & Cradle-grave & Driving distance & $\begin{array}{l}\text { NREL, EMEP-EEA } \\
\text { Guidebook, Aspen } \\
\text { Plus, ecoinvent, IPCC } \\
\text { guidline }\end{array}$ & SimaPro & CML & $\begin{array}{l}\text { Economic, system } \\
\text { expansion }\end{array}$ \\
\hline [38] & Hemp hurds & Spain & Cradle-grave & Driving distance & NREL, ecoinvent & ND & CML & Mass, economic \\
\hline [39] & Fiber sorghum & Italy & Cradle-grave & Input biomass & $\begin{array}{l}\text { Experimental data, } \\
\text { ecoinvent, IPCC } \\
\text { guidline }\end{array}$ & SimaPro & ReCiPe & ND \\
\hline [40] & Arundo donax $L$. & Italy & Cradle-grave & Driving distance & $\begin{array}{l}\text { Experimental data, } \\
\text { ecoinvent, IPCC } \\
\text { guidline }\end{array}$ & SimaPro & ReCiPe & $\begin{array}{l}\text { Energy, } \\
\text { economic }\end{array}$ \\
\hline [17] & $\begin{array}{l}\text { Wheat straw, forest } \\
\text { residue, poplar, and } \\
\text { miscanthus }\end{array}$ & UK & Cradle-gate & Ethanol product & $\begin{array}{c}\text { NREL, Aspen Plus, } \\
\text { ecoinvent, GEMIS } \\
\text { model }\end{array}$ & GaBi & CML & $\begin{array}{l}\text { Economic, system } \\
\text { expansion }\end{array}$ \\
\hline [41] & Cassava & Thailand & Cradle-gate & Ethanol product & Industry, ecoinvent & SimaPro & CML & Energy \\
\hline [42] & Switchgrass & Netherlands & Cradle-gate & Driving distance & NREL, ecoinvent & CMLCA & CML & $\begin{array}{l}\text { Energy, } \\
\text { economic }\end{array}$ \\
\hline [43] & Flax shives & Spain & Cradle-grave & Ethanol product & $\begin{array}{l}\text { NREL, on-site survey, } \\
\text { ecoinvent }\end{array}$ & CMLCA & CML & $\begin{array}{c}\text { Mass, } \\
\text { economic }\end{array}$ \\
\hline [44] & Alfalfa stems & Spain & Cradle-grave & Driving distance & NREL, ecoinvent & CMLCA & CML & Mass \\
\hline [45] & Bagasse & South Africa & Cradle-gate & Input biomass & $\begin{array}{c}\text { Industry, TEAM } \\
\text { database }\end{array}$ & TEAM & $\mathrm{CML}$ & ND \\
\hline [46] & Brassica carinata & Spain & Cradle-grave & $\begin{array}{l}\text { Driving distance, } \\
\text { ethanol product }\end{array}$ & $\begin{array}{l}\text { NREL, on-site survey, } \\
\text { ecoinvent, EIPRO } \\
\text { database }\end{array}$ & CMLCA & CML & not used \\
\hline [47] & Willow & Sweden & Cradle-gate & Input biomass & $\begin{array}{c}\text { NREL, ecoinvent, } \\
\text { on-site survey, Aspen } \\
\text { Plus }\end{array}$ & SimaPro & CML & Economic \\
\hline [48] & Eucalyptus & Spain & Cradle-grave & Driving distance & $\begin{array}{l}\text { NREL, ecoinvent, } \\
\text { on-site survey }\end{array}$ & CMLCA & CML & ND \\
\hline [49] & $\begin{array}{c}\text { Cassava straw, cassava } \\
\text { root }\end{array}$ & China & Cradle-gate & Ethanol product & NREL, Aspen Plus & $\mathrm{GaBi}$ & CML & ND \\
\hline
\end{tabular}


Table 1. Cont.

\begin{tabular}{|c|c|c|c|c|c|c|c|c|}
\hline $\begin{array}{l}\text { Reference/Authors and } \\
\text { Reference Number as } \\
\text { Required by Energies }\end{array}$ & Biomass Source & Location & System Boundary & Functional Unit & $\begin{array}{l}\text { Data Source/Type of } \\
\text { Collected Data }\end{array}$ & Software & LCIA Method/Type & Allocation Method \\
\hline [50] & Bagasse & India & Gate-gate & Input biomass & $\begin{array}{c}\text { EPA, NREL, Aspen } \\
\text { Plus, TEAM } \\
\text { databases }\end{array}$ & TEAM & CML, IPCC & ND \\
\hline [51] & Bagasse, Sugarcane & Brazil & Cradle-gate & Ethanol product & $\begin{array}{c}\text { Industry, NREL, } \\
\text { Aspen Plus }\end{array}$ & SimaPro & CML & Economic \\
\hline [52] & $\begin{array}{c}\text { Timber, recycled } \\
\text { newsprint }\end{array}$ & USA & Cradle-gate & Ethanol product & $\begin{array}{c}\text { Boustead model, } \\
\text { NREL, Aspen Plus, } \\
\text { USDA Forest Service } \\
\text { database }\end{array}$ & EFRAT & Ecoindicator & ND \\
\hline [53] & $\begin{array}{l}\text { Hay, agricultural and } \\
\text { forest wood waste }\end{array}$ & Canada & Cradle-grave & Driving distance & Ecoinvent & SimaPro & Ecoindicator & not used \\
\hline [54] & Bagasse & South Africa & Cradle-gate & Caloric value & Industry, ecoinvent & SimaPro & IMPACT 2002 & System expansion \\
\hline [55] & Willow & UK & Cradle-grave & Ethanol product & $\begin{array}{c}\text { NREL, GaBi } \\
\text { databases, Aspen } \\
\text { HYSYS }\end{array}$ & $\mathrm{GaBi}$ & EDIP & $\begin{array}{l}\text { System expansion, } \\
\text { energy }\end{array}$ \\
\hline [56] & $\begin{array}{l}\text { Maize grain, maize } \\
\text { stover, sugarcane, sugar } \\
\text { beet and wheat }\end{array}$ & USA, Brazil, France & Cradle-gate & Ethanol product & Ecoinvent & SimaPro & ReCiPe & Economic \\
\hline [57] & $\begin{array}{l}\text { Eastern redcedar, corn } \\
\text { grain }\end{array}$ & USA & Cradle-gate & Caloric value & $\begin{array}{c}\text { Ecoinvent, NREL, } \\
\text { Aspen Plus } \\
\text { SuperPro }\end{array}$ & SimaPro & $\begin{array}{c}\text { IMPACT 2002, } \\
\text { BEES+ }\end{array}$ & System expansion \\
\hline [58] & Sugarcane, bagasse & Brazil & Cradle-gate & Ethanol product & $\begin{array}{l}\text { Designer, CanaSoft } \\
\text { model, ecoinvent, } \\
\text { NREL }\end{array}$ & GaBi & $\begin{array}{l}\text { ILCD 2011, } \\
\text { Ecoindicator }\end{array}$ & Economic \\
\hline
\end{tabular}


In cases of using bioethanol for FFVs blending refinery, distribution points and final bioethanol product have been included by a few studies [24,30,32,34]. However, an ideal cradle-grave system boundary for bioethanol (as fuel) should include manufacturing, maintenance and disposal of a vehicle, road construction, as well as upstream information, e.g., chemicals, but only a small portion of papers argued that.

Around $78 \%$ of the reviewed studies (mostly used second-generation feedstock) considered energy as a co-product of bioethanol production and heat/electricity production in their system boundary. Shadbahr et al. [13] directly investigated boundary changes on LCA results by shifting the system boundary from the pre-treatment stage to the whole bioethanol production plant. This study shows that after extending the system boundaries, the indicator values of the most environmental impact and damage categories improved. The credit given to on-site steam and electricity generation from residues was the main reason for improved impact indicator values.

Functional unit (FU) is another important component of LCA methodology defined as a reference flow to signify the output of processes within the LCA. The definition of the functional unit (FU) related to the purpose of the study influences the interpretation of the results. Moreover, selecting an appropriate functional unit can illustrate the environmental performance of the system boundary in different aspects. González-García et al. [43] analyzed impact categories for two different functional units comparing E10 fuel with E85. According to their findings, LCA results are changed by choosing functional units for similar system boundaries.

In general, there is no uniformity among functional units presented in the reviewed studies. Typical functional units selected in the reviewed LCA studies are related to biomass input, land area to produce the dedicated input or final output (i.e., final product or service of the process). About $85 \%$ of the reviewed studies used functional units relevant to outputs like mass/volume of ethanol product or $\mathrm{km}$ of transportation. Figure 4 shows the number of reviewed papers with different functional units.

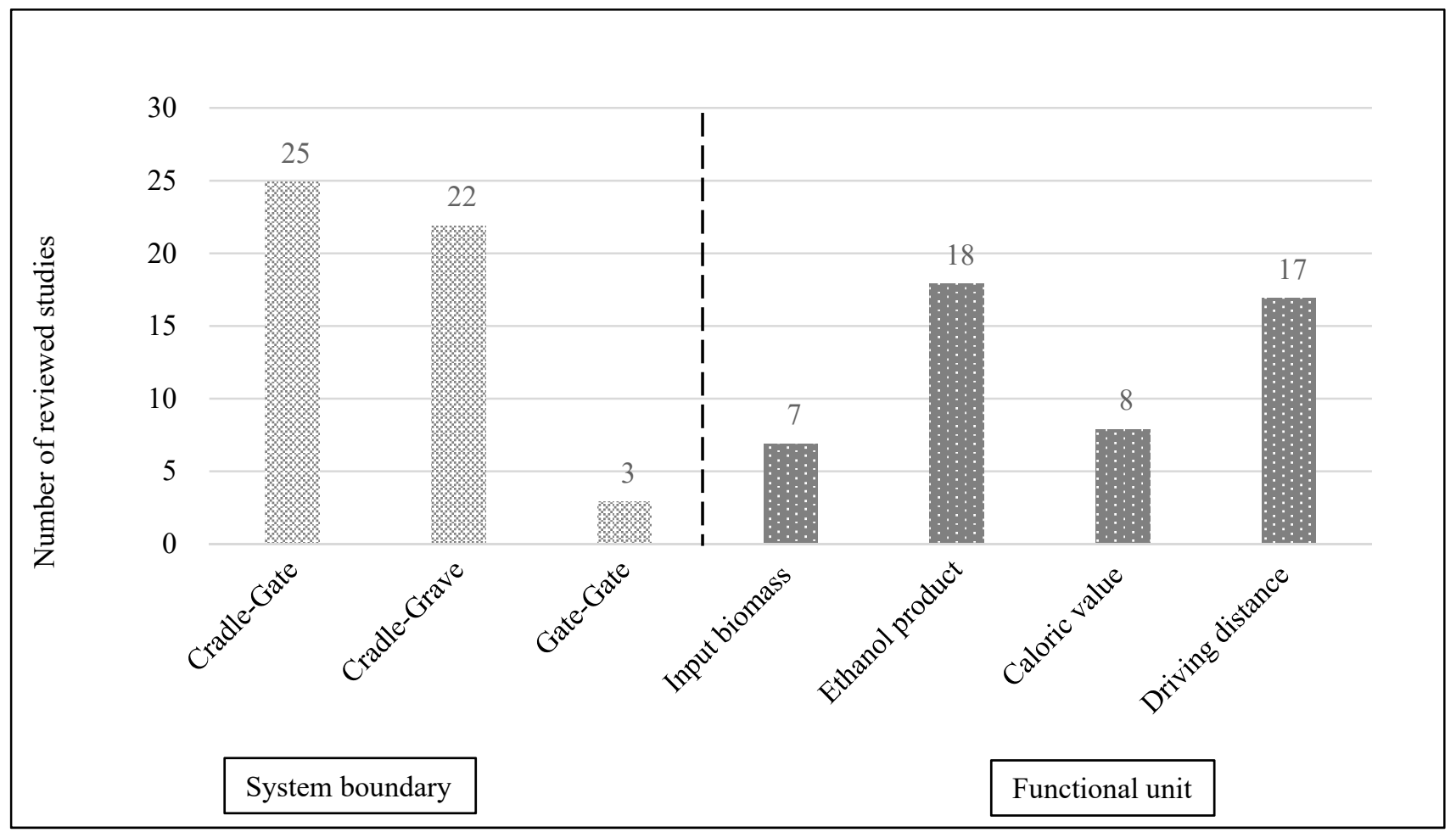

Figure 4. Number of reviewed papers in each selected functional unit and system boundary (some of studies chose more than one FU). 


\subsubsection{Life Cycle Inventory}

\section{Data Source}

Different data sources can be used for providing the inventories of an LCA study. The reviewed papers obtained data from industry, expert interviews, estimation and LCA software databases. Every study tried to have completeness of data by adding different datasets. Further, most of the studies compensated missing real data by data sets from literature, software databases and simulations. Data gathering from reports, models and simulations are widely used in the reviewed LCA studies. Table 2 gives an overview of the employed data sources used for life cycle inventory preparation in the reviewed studies.

Table 2. Data sources used for life cycle inventory stage of the reviewed bioethanol LCAs.

\begin{tabular}{ccc}
\hline Type of Collected Data & Data Source & Reference \\
\hline $\begin{array}{c}\text { Primary data for the agricultural activities } \\
\text { Emission data related to fossil fuel, combustion and }\end{array}$ & CanaSoft model & {$[58]$} \\
auxiliary materials production & GREET model & {$[20,22,27,29,33,35]$} \\
$\begin{array}{c}\text { Processes of bioethanol production } \\
\text { Transportation, ethanol and electricity production, } \\
\text { Agricultural data, facility and equipment design }\end{array}$ & Aspen Plus, SuperPro Designer & {$[14,15,17,31,33,47,49-52,55,57,58]$} \\
Emissions from capital goods production & NREL report & {$[7,8,13,15,17-19,22,30,32,33,37,38,42-$} \\
Emission from agricultural activities, fuel combustion & EIPRO database & $44,46-52,55,57,58]$ \\
and field operation & EMEP-EEA Guidebook & {$[7,26]$} \\
Emissions inventory & Boustead Model & {$[15]$} \\
Pesticide application emissions & PestLCI software & {$[52]$} \\
\hline
\end{tabular}

\subsubsection{Software Use}

There are different software tools available facilitating LCA compilation. As shown in Table 1 the reviewed studies used popular open-source or commercial software tools. SimaPro was the most used tool among all studies. Furthermore, some studies selected other software and tools such as TEAM or CMLCA to calculate environmental impacts. Moreover, in one paper authors connected inventory data with the Environmental Fate and Risk Assessment Tool (EFRAT) to assess eight impact categories [52]. Table 3 Shows the details of tools used by the reviewed studies.

Table 3. Life cycle assessment tools used in reviewed papers.

\begin{tabular}{|c|c|c|}
\hline Software & Developer & Country \\
\hline SimaPro & Pré-consultants & Netherland \\
\hline $\mathrm{GaBi}$ & PE International & Germany \\
\hline Umberto & Ifu Institute & Germany \\
\hline TEAM & Ecobilan-PricewaterhouseCoopers & France \\
\hline CMLCA & $\begin{array}{c}\text { Institute of Environmental Sciences (CML), } \\
\text { Leiden University }\end{array}$ & Netherland \\
\hline $\begin{array}{l}\text { EFRAT (Environmental Fate } \\
\text { and Risk Assessment Tool) }\end{array}$ & $\begin{array}{l}\text { Institute of Environmental Sciences (CML), } \\
\text { Leiden University } \\
\text { Department of Chemical Engineering and } \\
\text { Department of Environmental Engineering } \\
\text { at MichiganTech University }\end{array}$ & United States \\
\hline
\end{tabular}

\subsubsection{By-Product Credit and Allocation}

Most of the agricultural and industrial processes provide more than one output. Bioethanol production inevitably generates co-/by-products like animal feed, electricity, heat, etc. Hence, the breakdown of environmental loads between the main product and the 
co-product is important. Choosing a suitable allocation method for sharing environmental loads among final outputs is still the most debated aspect in LCA methodology [59].

The allocation method based on the value of energy, mass and economy are very popular. Almost $56 \%$ of the reviewed studies introduced the allocation approaches in their work. The remaining followed the system expansion approach, neglect allocation procedure or did not mention in the paper. Since each study chose its allocation approach based on the purpose of the study, a comparison of the reviewed papers is hardly possible. 12 cases of reviewed papers took multiple approaches into consideration recognizing the influence of allocation methods on the outcomes of their work. Figure 5 presents an overview of allocation method selected by the reviewed papers.

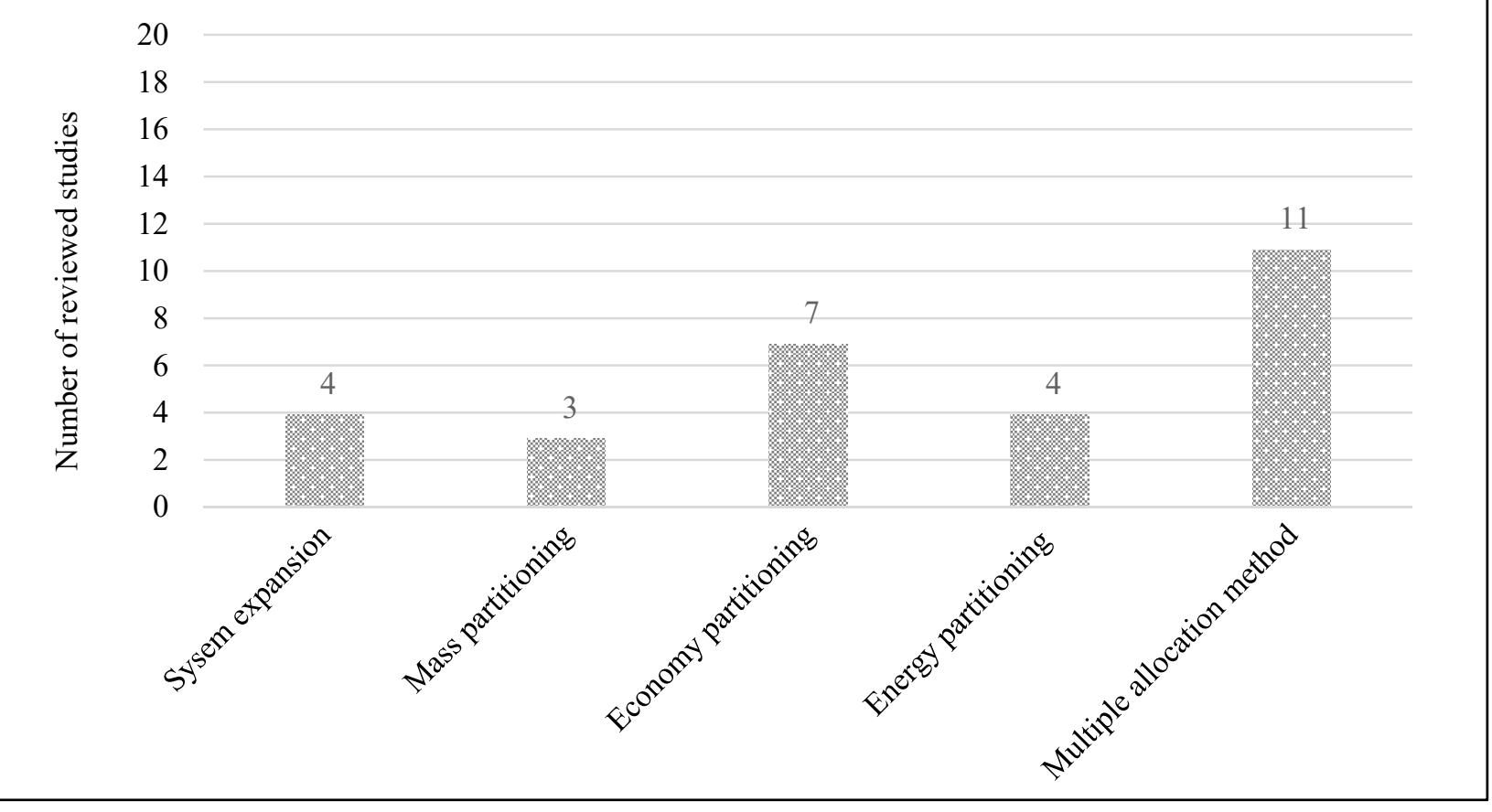

Figure 5. Different allocation methods in the reviewed papers.

\subsubsection{Applied Assessment Methods and Impact Categories}

Most of the LCAs of bioethanol are limited to the GWP as a single impact category. Here, only studies including a set of impact categories were considered. The review revealed that most studies preferred to omit the normalization and weighting step in the assessment stage. Only a few papers $(18 \%)$ presented the results after normalization and two studies applied weighting step.

The impact assessment methods used in the papers comprised both midpoint (problemoriented) and endpoint (damage-oriented) levels, as well as combined-methods in some cases. According to Figure 6, CML assessment method as a midpoint level method is most widely used, followed by EcoIndicator and ReCiPe.

Each applied assessment method covers a series of impact categories. Nevertheless, some of the studies just selected a few categories. Mostly acidification, eutrophication and ecotoxicity (air, water, terrestrial, marine or human) have been assessed. According to Figure $7,100 \%$ of the reviewed studies used a climate-related impact category when investigated the life cycle impacts of bioethanol. More than $50 \%$ of the studies evaluate two further impacts related to air pollution (i.e., ozone depletion, photochemical ozone creation). 


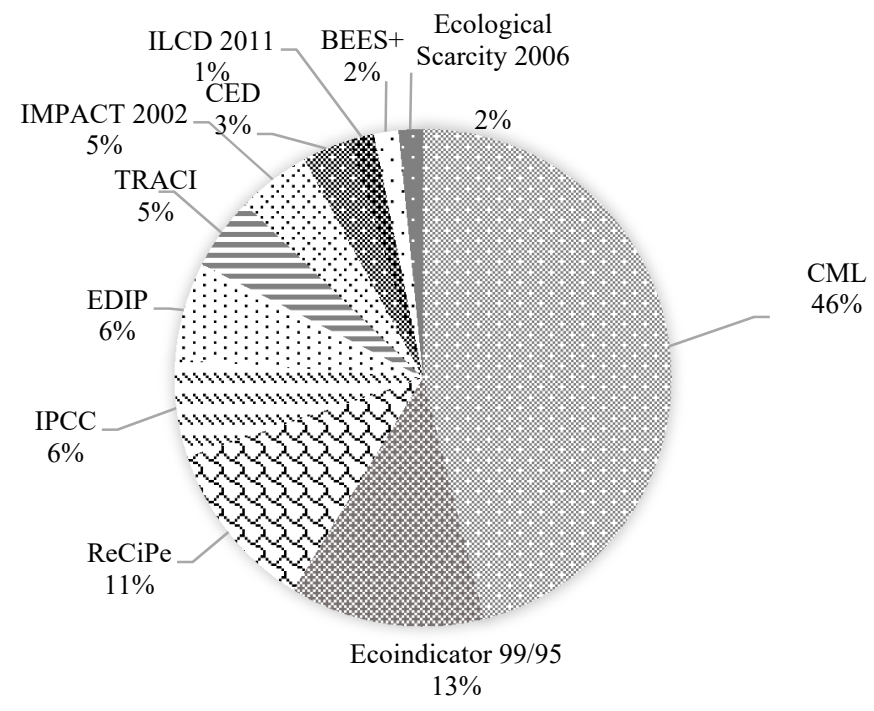

Figure 6. Share of most used assessment methods in previous LCA studies.

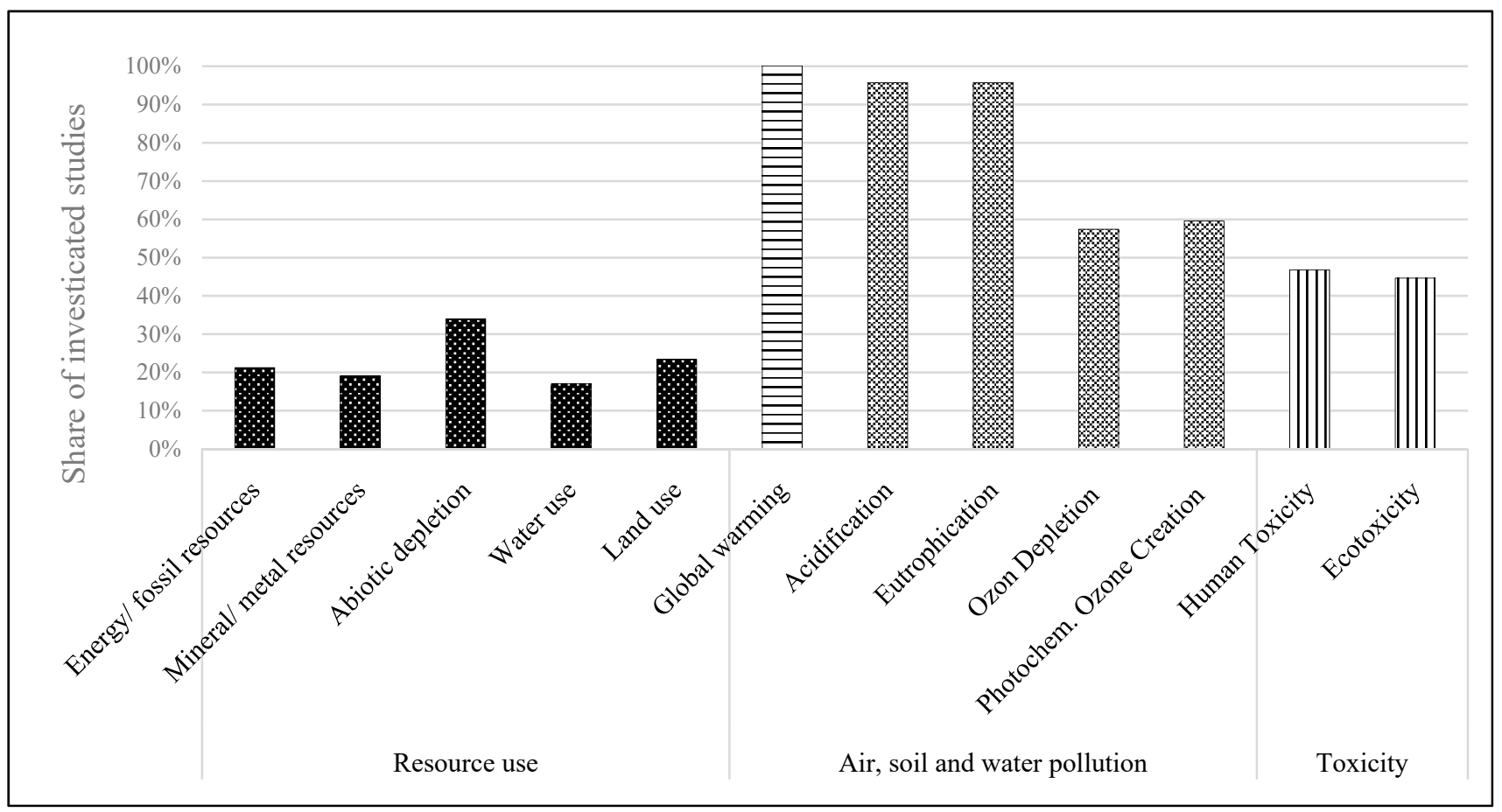

Figure 7. In each impact category, the proportion of studies that use the corresponding impact category is shown to the total number of all studies examined (in \%).

Around $80 \%$ of the reviewed paper reported that bioethanol production despite the reduction in global warming potential increases acidification, eutrophication and photochemical oxidant formation impact categories. The assessment of the depletion of fossil energy resources is done by $21 \%$ of the studies through CED or the respective impact category within the ReCiPe. Additionally, the portion of studies that also examine an impact category related to abiotic resource use is low. However, 16 out of the 48 studies, considered impacts from abiotic resource use. Of these, $34 \%$ deploy the impact category Abiotic Depletion Potential from the Dutch CML method. All studies analyzing the abiotic degradation potential showed that bioethanol production from biomass offers advantages in this category compared to conventional fossil-based gasoline. $19 \%$ of the analyzed 
studies also assessed the categories of mineral resource and metal depletion of ReCiPe and IMPACT 2002 methods.

Despite covering various categories by different assessment methods, some categories still receive poor attention. For instance, only a few studies consider the significant impact (directly/indirectly) of bioethanol production on land-use change and water consumption.

Approximately $17 \%$ of the studies investigate impact on water consumption, although this topic seems to be quite relevant for bio-based energy. This is probably mainly due to a lack of a methodological consensus for water use assessment in the context of LCA at the time most of the reviewed studies were published. There have been several attempts for water use assessment, but the first kind of approved method was just published in 2017 [60]. The studies reviewed here used ReCiPe for assessing the impact category water use.

Another very relevant impact category for the assessment of biofuels is land use. $23 \%$ of the reviewed studies assessed the impacts of land use. They mostly used impact categories are based on the Ecoindicator 99 and CML 2 Baseline 2000 methods. High impacts from land use were found when forests or grasslands are converted to biomass crops with low biofuel yield. Using waste materials or woody crops (e.g., from short rotation plantations) is more favorable in terms of land use impacts $[17,57]$. When comparing bio-based and fossil-based ethanol in terms of land use it would be advisable to prefer fossil-based fuels because there are always impacts from land use when switching to bio-based fuels [56].

Moreover, to pay more attention to land use impact category, Muñoz. et al. extended their research by adding novel midpoint impact categories on Biodiversity and Ecosystem Services [56]. The BES indicator comprises seven impact categories including biodiversity damage potential [61], climate regulation potential [62], biotic production potential [63], freshwater regulation potential, erosion regulation potential, water purification potential through physicochemical filtration and water purification potential through mechanical filtration [64] to consider relevant impacts to land occupation.

\subsubsection{Uncertainties}

Uncertainty analysis is included in the interpretation part of the LCA methodology to address the range of variation in the results. Most of the published papers have not included an uncertainty analysis. Among the reviewed LCA studies, 17\% of them (8 cases) examined the uncertainties on the results. They analyzed uncertainty by a Monte Carlo function that was included in the LCA software. In these cases, all input/output data from the upstream processes to usages phase in transportation were included $[39,57]$. The reviewed papers indicated that uncertainties occur due to a lack of data or data inaccuracy in industrial processes, methodological elements selection (e.g., system boundary, allocation, impact categories and characterization models), as well as feedstock preparation.

According to the review, 13 studies used simulation software to prepare data inventories. In this regard, there is a risk of estimating the impacts of processes in the conceptual design, pilot and laboratory rather than commercial scale because it is not easy to examine the efficiency of the technologies in the early stages [65]. Not only the maturity of technology but also the construction of equipment and facilities could be a source of data scarcity. Amongst reviewed studies there was only one study, which included material used for plant construction [35]. The influence of technology in the result uncertainty was taken into account by Borrion et al. [30]. They found that the level of technology development affects the reliability of data and the results.

The methodological choices are another potential cause for uncertainties in LCA results. However, the recent LCA studies attempt to cover more impact categories but in most of them impact categories such as human toxicity, biodiversity, land and water use are still under poor attention. The data gap due to employing literature and similar projects to collect data is unavoidable, especially due to geographical or temporal differences in particular when normalization is considered in the study. 
In our review, six of eight studies that included uncertainty analysis in their work, examined agricultural parameters to estimate the uncertainties. These studies showed that the gap of the data in the agricultural stage (e.g., land-use change, soil emissions, carbon cycle) contributes to uncertainties $[34,36,56]$.

\section{Conclusions}

In recent years, a wide range of LCA studies has been conducted to measure the sustainability of bioethanol production. As was mentioned, the differences in methodological assumptions, technical and agricultural issues applied by authors hampered the comparison of LCA outcomes. In other words, it is not possible to compare the influence of each component of the methodology on the results with a high certainty, because various technical and agricultural-related parameters are also involved in the results of studies. However, in this paper selected studies were reviewed to identify bottlenecks in LCA analysis in terms of the three aspects mentioned above.

- $\quad$ Reviewed papers show that studies results depend greatly on LCA methodology choices. Selection of allocation method, the definition of assessment method, system boundary and functional unit besides choosing effective impact categories change the results.

However, there is an increasing trend of considering a set of impact categories in LCIA stage rather than only GHG impact. On the other hand, the share of studies that examine impact categories relevant to land and water use is not significant. Therefore, future studies should focus more on ecosystem quality to clarify the potential impacts of bioethanol production on land-use changes including water resources.

A handful of reviewed papers considered uncertainty analysis. Some of them studied conceptual models which may not precisely represent the industrial system that would be built in the future; hence, the differences between data obtained from the simulation and actual industrial data makes the uncertainties. Besides, many other variables caused by methodological assumptions and agricultural issues are involved in a wide range of uncertainties. Attention needs to be placed on uncertainty analysis in LCA studies. Furthermore, the studies can reduce the potential uncertainties by reconsidering the key elements of LCA methodology such as FU, system boundary, as well as data collection process.

- Although most authors added the feedstock production and processing into the system boundary, less than half of them considered agrochemicals' production. Given the important role of chemicals, such as fertilizers, pesticides in increasing acidification, eutrophication, land-use change and carcinogens, it seems necessary to include the production of those chemicals in LCA studies. Moreover, carrying out LCA analysis on the bioethanol from new generation of feedstock with less chemicals requirements or less agricultural practices would be useful to estimate and compare the relevant impacts with conventional feedstock.

- From the technical point of view, pre-treatment was the most considered process in the studies with a high potential for changing LCA results. As reviewed papers showed that the modified pre-treatment methods lead to environmental savings, because pre-treatment plays an important role in the next processes. However, advanced pre-treatment technologies with optimization of inputs and outputs in the bioethanol production can decrease the environmental impacts; the other processes of production also need to be considered. Furthermore, in most circumstances, studies neglected the production, transportation and use of the chemicals, nutrients, enzymes, which are the inputs for bioethanol production processes. This aspect needs to be investigated more by future studies. 
Author Contributions: Conceptualization, T.S.A., K.G. and M.K.; methodology, T.S.A., M.K. and K.G.; formal analysis, T.S.A. and A.R.; data curation, T.S.A. and A.R.; visualization, T.S.A. and A.R.; resources, T.S.A. and A.R.; writing-original draft preparation, T.S.A. and A.R.; writing-review and editing, T.S.A., K.G., A.R. and M.K.; supervision, M.K. and K.G. All authors have read and agreed to the published version of the manuscript.

Funding: This research was funded by the grant "Excellence initiative-research university" for the AGH University of Science and Technology, no. 16.16.150.545.

Institutional Review Board Statement: Not applicable.

Informed Consent Statement: Not applicable.

Data Availability Statement: Not applicable.

Conflicts of Interest: The authors declare no conflict of interest.

\section{Acronyms}

$\begin{array}{ll}\text { LCA } & \text { Life Cycle Assessment } \\ \text { FU } & \text { Functional Unit } \\ \text { FFVs } & \text { Flex Fuel Vehicles } \\ \text { 1G } & \text { First generation } \\ \text { 2G } & \text { Second generation } \\ \text { CED } & \text { Cumulative Energy Demand } \\ \text { CMLCA } & \text { Chain Management by Life Cycle Assessment } \\ \text { LUC } & \text { Land Use Change } \\ \text { GHG } & \text { Greenhouse Gases } \\ \text { E10 } & \text { Mixture of } 10 \% \text { ethanol and 90\% gasoline by volume } \\ \text { E85 } & \text { Mixture of 85\% ethanol and 15\% gasoline by volume } \\ \text { ISO } & \text { International Standards Organization } \\ \text { BES } & \text { Biodiversity and Ecosystem Services } \\ \text { GWP } & \text { Global Warming Potential } \\ \text { IPCC } & \text { Intergovernmental Panel on Climate Change } \\ \text { EDIP } & \text { Environmental Design of Industrial Products } \\ \text { CML } & \text { Institute of Environmental Sciences of the Faculty of Science of Leiden University } \\ \text { BEES+ } & \text { Building for Environmental and Economic Sustainability } \\ \text { ILCD } & \text { The International Reference Life Cycle Data System } \\ \text { TRACI } & \text { Tool for the Reduction and Assessment of Chemical and Other Environmental Impacts } \\ \text { LCIA } & \text { Life Cycle Impact Assessment }\end{array}$

\section{References}

1. Edenhofer, O.; Pichs-Madru, R.; Sokona, Y.; Seyboth, K.; Matschoss, P.; Kadner, S.; Zwickel, T.; Eickemeier, P.; Hansen, G.; Schloemer, S.; et al. IPCC Special Report of the Intergovernmental Panel on Climate Change; Cambridge University Press: New York, NY, USA, 2012.

2. Directive (EU) 2018/2001 of the European Parliment and of the Council. 2018. Available online: https://eur-lex.europa.eu (accessed on 28 February 2021).

3. Renewable Energy Prospects for the European Union. 2018. Available online: https:/ /www.irena.org/ (accessed on 28 February 2021).

4. U.S. Department of Energy. Available online: https:/ / afdc.energy.gov/data/ (accessed on 28 February 2021).

5. ePURE. European Renewable Ethanol-Key Figures. 2017. Available online: https://www.epure.org/ (accessed on 28 February 2021).

6. Vohra, M.; Manwar, J.; Manmode, R.; Padgilwar, S.; Patil, S. Bioethanol production: Feedstock and current technologies. J. Environ. Chem. Eng. 2014, 2, 573-584. [CrossRef]

7. Luo, L.; van der Voet, E.; Huppes, G.; Udo de Haes, H.A. Allocation issues in LCA methodology: A case study of corn stover-based fuel ethanol. Int J. Life Cycle Assess. 2009, 14, 529-539. [CrossRef]

8. González-García, S.; Moreira, M.T.; Feijoo, G. Comparative environmental performance of lignocellulosic ethanol from different feedstocks. Renew. Sustain. Energy Rev. 2010, 14, 2077-2085. [CrossRef]

9. ISO (International Organization for Standardization). Environmental Management_Life Cycle Assessment_Principles and Framework; Standard ISO14040; ISO: Geneva, Switzerland, 2006. 
10. ISO (International Organization for Standardization). Environmental Management_Life Cycle Assessment-Requirements and Guidelines; Standard ISO 14044; ISO: Geneva, Switzerland, 2006.

11. Hauschild, M.Z.; Rosenbaum, R.K.; Olsen, S.I. Life Cycle Assessment: Theory and Practice; Springer: Cham, Switzerland, 2018.

12. da Costa Sousa, L.; Chundawat, S.P.S.; Balan, V.; Dale, B.E. 'Cradle-to-grave' assessment of existing lignocellulose pretreatment technologies. Curr. Opin. Biotechnol. 2009, 20, 339-347. [CrossRef]

13. Shadbahr, J.; Zhang, Y.; Khan, F. Life cycle assessment of bioethanol production from woodchips with modifications in the pretreatment process. Appl. Biochem. Biotechnol. 2015, 175, 1080-1091. [CrossRef]

14. Guo, M.; Littlewood, J.; Joyce, J.; Murphy, R. The environmental profile of bioethanol produced from current and potential future poplar feedstocks in the EU. Green Chem. 2014, 16, 4680-4695. [CrossRef]

15. Wang, L.; Littlewood, J.; Murphy, R.J. Environmental sustainability of bioethanol production from wheat straw in the UK. Renew. Susaint. Energy Rev. 2013, 28, 715-725. [CrossRef]

16. Ntihuga, J.N.; Senn, T.; Gschwind, P.; Kohlus, R. Estimating energy and eco-balances for continuous bio-ethanol production using a Blenke cascade system. Energies 2013, 6, 2065-2083. [CrossRef]

17. Falano, T.; Jeswani, H.K.; Azapagic, A. Assessing the environmental sustainability of ethanol from integrated biorefineries. Biotechnol. J. 2014, 9, 753-765. [CrossRef]

18. González-García, S.; Moreira, M.T.; Feijoo, G.; Murphy, R.J. Comparative life cycle assessment of ethanol production from fast-growing wood crops (black locust, eucalyptus and poplar). Biomass Bioenergy 2012, 39, 378-388. [CrossRef]

19. Pieragostini, C.; Aguirre, P.; Mussati, M.C. Life cycle assessment of corn-based ethanol production in Argentina. Sci. Total Environ. 2014, 472, 212-225. [CrossRef] [PubMed]

20. Zhang, J.; Jia, C.; Wu, Y.; Xia, X.; Xi, B.; Wang, L.; Zhai, Y. Life cycle energy efficiency and environmental impact assessment of bioethanol production from sweet potato based on different production modes. PLoS ONE 2017, 12, e0180685. [CrossRef] [PubMed]

21. Mayer, F.D.; Brondani, M.; Aita, B.C.; Hoffmann, R.; Lora, E.S. Environmental and energy assessment of small scale ethanol fuel production. Energy Fuels 2015, 29, 6704-6716. [CrossRef]

22. Kim, S.; Dale, B.E. Life cycle assessment of fuel ethanol derived from corn grain via dry milling. Bioresour. Technol. 2008, 99, 5250-5260. [CrossRef]

23. Cavalett, O.; Chagas, M.F. Comparative LCA of ethanol versus gasoline in Brazil using different LCIA methods. Int. J. Life Cycle Assess. 2013, 647-658. [CrossRef]

24. Buchspies, B.; Kaltschmitt, M. Life cycle assessment of bioethanol from wheat and sugar beet discussing environmental impacts of multiple concepts of co-product processing in the context of the European Renewable Energy Directive context of the European Renewable Energy Directive. Biofuels 2016, 7, 141-153. [CrossRef]

25. Ometto, A.R.; Hauschild, M.Z. Lifecycle assessment of fuel ethanol from sugarcane in Brazil. Int. J. Life Cycle Assess. 2009, $236-247$. [CrossRef]

26. Luo, L.; Van Der Voet, E.; Huppes, G. Life cycle assessment and life cycle costing of bioethanol from sugarcane in Brazil. Renew. Sustain. Energy Rev. 2008, 13, 1613-1619. [CrossRef]

27. Wang, M.; Shi, Y.; Xia, X.; Li, D.; Chen, Q. Life-cycle energy efficiency and environmental impacts of bioethanol production from sweet potato. Bioresour. Technol. 2013, 133, 285-292. [CrossRef]

28. Foteinis, S.; Kouloumpis, V.; Tsoutsos, T. Life cycle analysis for bioethanol production from sugar beet crops in Greece. Energy Policy 2011, 39, 4834-4841. [CrossRef]

29. Wang, M.; Chen, Y.; Xia, X.; Li, J.; Liu, J. Energy efficiency and environmental performance of bioethanol production from sweet sorghum stem based on life cycle analysis. Bioresour. Technol. 2014, 163, 74-81. [CrossRef] [PubMed]

30. Borrion, A.L.; Mcmanus, M.C.; Hammond, G.P. Environmental life cycle assessment of bioethanol production from wheat straw. Biomass Bioenergy 2012, 47, 9-19. [CrossRef]

31. Aguilar-Sánchez, P.; Navarro-Pineda, F.S.; Sacramento-Rivero, J.C.; Barahona-Pérez, L.F. Life-cycle assessment of bioethanol production from sweet sorghum stalks cultivated in the state of Yucatan, Mexico. Clean. Technol. Environ. 2018, 20, 1685-1696. [CrossRef]

32. Daylan, B.; Ciliz, N. Life cycle assessment and environmental life cycle costing analysis of lignocellulosic bioethanol as an alternative transportation fuel. Renew. Energy 2016, 89, 578-587. [CrossRef]

33. Budsberg, E.; Rastogi, M.; Puettmann, M.E.; Caputo, J.; Balogh, S.; Volk, T.A.; Gustafson, R.; Johnson, L. Life-cycle assessment for the production of bioethanol from willow biomass crops via biochemical conversion. For. Prod. J. 2012, 62, 305-313. [CrossRef]

34. Cherubini, F.; Jungmeier, G. LCA of a biorefinery concept producing bioethanol, bioenergy, and chemicals from switchgrass. Int. J. Life Cycle Assess. 2010, 15, 53-66. [CrossRef]

35. Murphy, C.W.; Kendall, A. Life cycle analysis of biochemical cellulosic ethanol under multiple scenarios. Glob. Chang. Biol. Bioenergy 2015, 7, 1019-1033. [CrossRef]

36. Cherubini, F.; Ulgiati, S. Crop residues as raw materials for biorefinery systems-A LCA case study. Appl. Energy 2010, 87, 47-57. [CrossRef]

37. González-García, S.; Gasol, C.M.; Gabarrell, X.; Rieradevall, J.; Moreira, M.T.; Feijoo, G. Environmental profile of ethanol from poplar biomass as transport fuel in Southern Europe. Renew. Energy 2010, 35, 1014-1023. [CrossRef] 
38. González-García, S.; Luo, L.; Moreira, M.T.; Feijoo, G.; Huppes, G. Life cycle assessment of hemp hurds use in second generation ethanol production. Biomass Bioenergy 2012, 36, 268-279. [CrossRef]

39. Forte, A.; Zucaro, A.; Fagnano, M.; Fierro, A. Potential environmental impact of bioethanol production chain from fiber sorghum to be used in passenger cars. Sci. Total Environ. 2017, 598, 365-376. [CrossRef]

40. Zucaro, A.; Forte, A.; Basosi, R.; Fagnano, M.; Fierro, A. Life Cycle Assessment of second generation bioethanol produced from low-input dedicated crops of Arundo donax L. Bioresour. Technol. 2016, 219, 589-599. [CrossRef] [PubMed]

41. Papong, S.; Malakul, P. Life-cycle energy and environmental analysis of bioethanol production from cassava in Thailand. Bioresour. Technol. 2010, 101, S112-S118. [CrossRef] [PubMed]

42. Bai, Y.; Luo, L.; van der Voet, E. Life cycle assessment of switchgrass-derived ethanol as transport fuel. Int. J. Life Cycle Assess. 2010, 15, 468-477. [CrossRef]

43. González-García, S.; Luo, L.; Moreira, M.T.; Feijoo, G.; Huppes, G. Life cycle assessment of flax shives derived second generation ethanol fueled automobiles in Spain. Renew. Sustain. Energy Rev. 2009, 13, 1922-1933. [CrossRef]

44. González-García, S.; Moreira, M.T.; Feijoo, G. Environmental performance of lignocellulosic bioethanol production from Alfalfa stems. Biofuel Bioprod. Biorefin. 2010, 4, 118-131. [CrossRef]

45. Botha, T.; von Blottnitz, H. A comparison of the environmental benefits of bagasse-derived electricity and fuel ethanol on a life-cycle basis. Energy Policy 2006, 34, 2654-2661. [CrossRef]

46. González-García, S.; Gasol, C.M.; Gabarrell, X.; Rieradevall, J.; Moreira, M.T.; Feijoo, G. Environmental aspects of ethanol-based fuels from Brassica carinata: A case study of second generation ethanol. Renew. Sustain. Energy Rev. 2009, 13, 2613-2620. [CrossRef]

47. González-García, S.; Iribarren, D.; Susmozas, A.; Dufour, J.; Murphy, R.J. Life cycle assessment of two alternative bioenergy systems involving Salix spp. biomass: Bioethanol production and power generation. Appl. Energy 2012, 95, 111-122. [CrossRef]

48. González-García, S.; Moreira, M.T.; Feijoo, G. Environmental aspects of eucalyptus based ethanol production and use. Sci. Total Environ. 2012, 438, 1-8. [CrossRef]

49. Lyu, H.; Yang, S.; Zhang, J.; Feng, Y.; Geng, Z. Impacts of utilization patterns of cellulosic C5 sugar from cassava straw on bioethanol production through life cycle assessment. Bioresour. Technol. 2021, 323, 124586. [CrossRef] [PubMed]

50. Kadam, K.L. Environmental benefits on a life cycle basis of using bagasse-derived ethanol as a gasoline oxygenate in India. Energy Policy 2002, 30, 371-384. [CrossRef]

51. Dias, M.O.S.; Junqueira, T.L.; Cavalett, O.; Cunha, M.P.; Jesus, C.D.F.; Rossell, C.E.V.; Filho, R.M.; Bonomi, A. Integrated versus stand-alone second generation ethanol production from sugarcane bagasse and trash. Bioresour. Technol. 2012, 103, 152-161. [CrossRef]

52. Kemppainen, A.J.; Shonnard, D.R. Comparative life-cycle assessments for biomass-to-ethanol production from different regional feedstocks. Biotechnol. Prog. 2005, 21, 1075-1084. [CrossRef] [PubMed]

53. Fu, G.Z.; Chan, A.W.; Minns, D.E. Life cycle assessment of bio-ethanol derived from cellulose. Int. J. Life Cycle Assess. 2003, 8, 137-141. [CrossRef]

54. Melamu, R.; von Blottnitz, H. 2nd Generation biofuels a sure bet? A life cycle assessment of how things could go wrong. J. Clean. Prod. 2011, 19, 138-144. [CrossRef]

55. Stephenson, A.L.; Dupree, P.; Scott, S.A.; Dennis, J.S. The environmental and economic sustainability of potential bioethanol from willow in the UK. Bioresour. Technol. 2010, 101, 9612-9623. [CrossRef]

56. Muñoz, I.; Flury, K.; Jungbluth, N.; Rigarlsford, G.; Milà i Canals, L.; King, H. Life cycle assessment of bio-based ethanol produced from different agricultural feedstocks. Int. J. Life Cycle Assess. 2014, 19, 109-119. [CrossRef]

57. Olukoya, I.A.; Ramachandriya, K.D.; Wilkins, M.R.; Aichele, C.P. Life cycle assessment of the production of ethanol from eastern redcedar. Bioresour. Technol. 2014, 173, 239-244. [CrossRef]

58. Maga, D.; Thonemann, N.; Hiebel, M.; Sebastião, D.; Lopes, T.F.; Fonseca, C.; Gírio, F. Comparative life cycle assessment of firstand second-generation ethanol from sugarcane in Brazil. Int. J. Life Cycle Assess. 2019, 24, 266-280. [CrossRef]

59. Cherubini, F.; Strømman, A.H.; Ulgiati, S. Influence of allocation methods on the environmental performance of biorefinery products-A case study. Resour. Conserv. Recycl. 2011, 55, 1070-1077. [CrossRef]

60. Boulay, A.M.; Bare, J.; Benini, L.; Berger, M.; Lathuillière, M.J.; Manzardo, A.; Margni, M.; Motoshita, M.; Núñez, M.; Pastor, A.V.; et al. The WULCA consensus characterization model for water scarcity footprints: Assessing impacts of water consumption based on available water remaining (AWARE). Int. J. Life Cycle Assess. 2018, 23, 368-378. [CrossRef]

61. de Baan, L.; Alkemade, R.; Koellner, T. Land use impacts on biodiversity in LCA: A global approach. Int. J. Life Cycle Assess. 2013, 18, 1216-1230. [CrossRef]

62. Müller-wenk, R.; Brandão, M. Climatic impact of land use in LCA—Carbon transfers between vegetation/soil and air. Int. J. Life Cycle Assess. 2010, 15, 172-182. [CrossRef]

63. Brandão, M.; Milà i Canals, L. Global characterisation factors to assess land use impacts on biotic production. Int. J. Life Cycle Assess. 2013, 18, 1243-1252. [CrossRef]

64. Saad, R.; Koellner, T.; Margni, M. Land use impacts on freshwater regulation, erosion regulation, and water purification: A spatial approach for a global scale level. Int. J. Life Cycle Assess. 2013, 18, 1253-1264. [CrossRef]

65. Spatari, S.; Bagley, D.M.; MacLean, H.L. Life cycle evaluation of emerging lignocellulosic ethanol conversion technologies. Bioresour. Technol. 2010, 101, 654-667. [CrossRef] [PubMed] 